\title{
ENFOQUES CONCEPTUALES PARA INTERPRETAR LA PROBLEMÁTICA DEL DESARROLLO SOSTENIBLE
}

\author{
Vladímir M. Davydov \\ Miembro correspondiente de la Academia de Ciencias de Rusia \\ Doctor titular (Economía), prof. (davydov@ilaran.ru) \\ Director académico del ILA ACR
}

Instituto de Latinoamérica de la Academía de Ciencias de Rusia (ILA ACR)

B. Ordynka, 21/16, Moscú, 115035, Federación de Rusia

Jefe de la Cátedra de la Facultad de Economía

Universidad Rusa de la Amistad de los Pueblos (RUDN Universidad)

Calle Miklukho-Maklaya, 6, Moscú, 117198, Federación de Rusia

Recibido el 15 de julio de 2020

Aceptado el 16 de octubre de 2020

DOI: $10.37656 / \mathrm{s} 20768400-2020-4-01$

Resumen. La problemática del desarrollo sostenible ha pasado a ser uno de los temas predominantes (por no decir central) de la actual narrativa internacional en el ámbito publicístico y sociológico. Tan solo la pandemia COVID-19 ha podido relegarlo a un segundo plano. La situación existente en los países latinoamericanos o ibéricos no difiere del contexto mundial. El momento crucial de la problemática del desarrollo sostenible en la percepción social fue el consenso conformado en 2015 en torno al Acuerdo de París y a la resolución de la Asamblea General de la ONU sobre los objetivos del desarrollo sostenible hasta 2030. Entretanto, en la interpretación de dichos objetivos y en la formulación de la propia concepción del desarrollo sostenible persisten no pocas ambigüedades que imposibilitan que la comunidad mundial llegue a decisiones concordadas sobre medidas y actos conjuntos rumbo a superar los pecados del desarrollo "insostenible". El autor del artículo ofrece una interpretación perfeccionada (a su ver) de la concepción del desarrollo sostenible asentada en un enfoque 
integral tanto de la determinación como de los resultados del desarrollo de la economía de la sociedad y del Estado moderno.

Palabras clave: concepción del desarrollo sostenible, enfoque integral de la determinación del proceso de desarrollo, condicionamiento antropógeno de la crisis ecológica global

\title{
CONCEPTUAL APPROACHES TO SUSTAINABLE DEVELOPMENT PROBLEMATIC
}

\author{
Vladimir M. Davydov \\ Corresponding member of the Russian Academy of Sciences (RAS) \\ Dr.Sci.(Economy), prof. (davydov@ilaran.ru) \\ Academic Director of ILA RAS
}

Institute of Latin American Studies, Russian Academy of Science (ILA RAS)

21/16, B. Ordynka, Moscow, 115035, Russian Federation

Head of the Department of the Faculty of Economics

Peoples' Friendship University of Russia (RUDN University)

Miklujo-Maklaya, 6, Moscow, 117198, Russian Federation

Received on July 15, 2020

Accepted on October 16, 2020

DOI: $10.37656 / \mathrm{s} 20768400-2020-4-01$

Abstract. The sustainable development issues have become one of the mainstream trends (possibly, the central topic) of the current international discourse in the field of social sciences and political journalism. Only COVID-19 pandemic could challenge its relevance. In this aspect, the situation in the Latin American or Iberian countries shows no difference from the world context. The consensus reached in 2015 as the Paris Agreement on Climate Change and the Sustainable Development Goals to be achieved by the year 2030 set by the United Nations General Assembly, became the pivotal moment in the public perception of the sustainable development subject. However, several inconsistencies remaining both in the interpretation of said goals and the definition of the sustainable development conception, prevent the world community from making concurrent decisions on joint steps and actions intended to overcome the defects of the unsustainable development. The author of this paper offers an improved (in his opinion) version of the sustainable development conception based on the 
Enfoques conceptuales para interpretar la problemática del desarrollo sostenible

comprehensive approach both to the determination and results of the economy, society and contemporary State development.

Keywords: Sustainable development conception, comprehensive approach to development process determination, anthropogenic factor of the global ecological crises

\title{
КОНЦЕПТУАЛЬНЫЕ ПОДХОДЫ К ПРОБЛЕМАТИКЕ УСТОЙЧИВОГО РАЗВИТИЯ
}

\author{
Владимир Михайлович Давыдов \\ Член-корр.PAH, (davydov@ilaran.ru) \\ Научный руководитель ИЛА РАН \\ Институт Латинской Америки РАН \\ РФ. 115035, Москва, Б. Ордынка 21/16
}

Заведующий кафедрой экономического факультета РУДН

РФ, 117198, Москва, ул. Миклухо-Маклая, д. 6

Статья получена 15 июля 2020 г.

Статья принята 16 октября 2020 г.

DOI: $10.37656 / \mathrm{s} 20768400-2020-4-01$

Аннотация. Проблематика устойчивого развития стала одной из доминирующих (если не иентральной) тем современного международного нарратива в его обществоведческой $и$ публииистической ипостаси. Потеснить ее смогла лишь пандемия COVID-19. Ситуация в латиноамериканских или иберийских странах в этом смысле не отличается от общемирового контекста.

Поворотным моментом в общественном восприятии проблематики устойчивого развития стал консенсус, сложившийся в 2015 году вокруг Парижского соглашения по климату и резолючии Генеральной ассамблеи ООН относительно иелей устойчивого развития на период до 2030 года. Между тем в трактовке этих иелей и в формулировании самой конщепџии устойчивого развития остается немало разночтений, которые мешают выходу мирового сообщества на согласованные решения по поводу совместных мер и действий на пути к преодолению пороков «неустойчивого» развития.Автор статьи предлагает усовершенствованную (на его взгляд) трактовку концепции 
устойчивого развития, основанную на интегральном подходе $u \kappa$ детерминации, и к результатам развития экономики, общества и современного государства.

Ключевые слова: концепџия устойчивого развития, интегральный подход $\kappa$ детерминации процесса развития, антропогенная обусловленность глобального экологического кризиса

La percepción moderna de la idea del desarrollo sostenible no cabe, desde luego, en la noción literal de "sostenibilidad". La polémica al respecto es contraproducente. Ella, esta comprensión literal, está reñida de facto con la intelección del desarrollo como tal. $\mathrm{Si}$, por supuesto, no se reduce a una reproducción simple $\mathrm{o}$, incluso, en reducción. De suyo se entiende que el desarrollo siempre pasa por una alteración del equilibrio y un avance hacia el siguiente hito en una nueva espira. Conque, "non stop". Correspondientemente, la sostenibilidad respecto al desarrollo puede ser únicamente relativa. La consecución o la garantía del desarrollo sostenible no puede reducirse a cierta "estación de término". Se trata de una especie de horizonte, horizonte del planteamiento del siguiente objetivo. Es aplicable solamente a la situación de una determinada etapa. Y debe rectificarse acorde con sus resultados y las expectativas de la siguiente. En este sentido el concepto de los objetivos del desarrollo sostenible (ODS) no puede reducirse a cierta verdad abstracta absoluta. Está históricamente condicionado por "su" tiempo y es concreto en este sentido.

En este artículo veo como mi tarea intentar evaluar el significado y contenido de la concepción del desarrollo sostenible a la luz del cambio sustancial del contexto internacional de los últimos tiempos. No me propongo profundizar en una problemática meramente ecológica [1], consciente de mi escasez profesional en este terreno. Me pongo principalmente a abordar otra cosa: aquellos aspectos del tema 
en examen que llevan la concepción a su calidad integral, incluyendo su connotación económica, social y, en cierto grado, política.

$$
* * *
$$

Según parece, en una forma simplificada se entiende por "desarrollo sostenible" tal tipo de movimiento histórico progresivo de la economía y la sociedad, tal modelo de su organización institucional y actitud hacia el medio ambiente que impiden satisfacer las necesidades de hoy a expensas del futuro. En otros términos, el ideal del desarrollo sostenible en un cierto sentido se presenta como negación del desarrollo a crédito ante el futuro ( $\mathrm{y}$ ante las generaciones del porvenir). Tal interpretación se expuso en las conclusiones de la Comisión Brundtland [2, p. 11]. Además, es obvio que el propio ideal no puede ser constante, dado una vez y para siempre. Es movible y variable por su contenido a medida que pase a otros hitos, más altos, de su desarrollo, a medida de la modificación del mecanismo de reproducción. Correlativa e inevitablemente, debe cambiar la política para garantizar el desarrollo sostenible, la configuración de sus prioridades, la tecnología de acciones de adaptación y protección. Por fin, si en el proceso de adaptación es alcanzable en principio una transición masiva al uso de recursos renovables, entonces, a la vez, en una perspectiva previsible es poco probable que surja la amenaza de exclusión total de recursos no renovables de la circulación económica. Por cierto que tendrá lugar su agotamiento, lo que exigirá sin falta una racionalización estricta de su consumo. $\mathrm{Y}$, probablemente, su sustitución sistemática, en particular por sus análogos naturales. De igual manera debe evolucionar el esquema de producción, distribución y consumo. 
Conque, queda evidente que la matriz del desarrollo sostenible debe ser adaptativamente modificable. Pero la cuestión consiste también en otro asunto.. El desarrollo sostenible tiene diferente escala, diferente dimensión. Puede ser local, nacional, regional (macroregional) y global. Por eso, es menester ver y comprender tal diferencia. Empero, con todo eso, no se debe olvidar que se trata de los "vasos comunicantes".

Por desgracia, la situación en la economía mundial a inicios de los años 2020 no era de las mejores. La dinámica de la producción y el comercio había bajado a su mínimo en las siete últimas décadas. Esto se advertía palmariamente en la lectura de datos estadísticos a largo plazo, tanto en la dimensión mundial como en la regional. La financialización sustraía capital productivo y lo remitía al sector especulativo. En la élite política y de negocios prevalecían los presentimientos de mal agüero. Pocos eran los que excluían la probabilidad de desestabilización crítica de la economía mundial en una perspectiva a corto plazo. Así se caracterizaba y se percibía la situación antes de la pandemia del coronavirus. Post factum los matemáticos pueden decir que la situación había empeorado en un orden de magnitud. En particular, si tratamos de Rusia, que sufrió un grave impacto después de la negativa, a inicios de 2020, a limitar la extracción de petróleo según la fórmula de OPEC + . Al aplicarse a nivel local, la orientación al desarrollo sostenible no puede prescindir de garantías efectivas a nivel internacional, en el fondo es condición sine qua non. Y esto quiere decir: entre las más importantes garantías internacionales se ubica y se ubicará el control de armas, sobre todo armas de exterminio en masa, la limitación de la inercia de militarización y una contribución eficaz a la paz. Y, correlativamente, una garantía cabal de la seguridad estratégica. Esto es tanto más 
importante para nosotros hoy día cuanto que en EE.UU. ha venido a prevalecer un rumbo a la confrontación en los quehaceres internacionales. Al respecto, los prestigiosos analistas hablan, y no sin razón, de una segunda edición de la "guerra fría". Tal es el sentido del informe que el académico Serguey Rógov presentó en la asamblea general de la Academia de Ciencias de Rusia el 13 de noviembre del año pasado. Washington se retracta de sus compromisos relativos a los tratados clave que durante decenios garantizaban el equilibrio estratégico de potenciales bélicos y la seguridad estratégica. El vacío emergente provoca una nueva carrera armamentista que gravita fuertemente sobre la voluntad política de los Estados líderes $\mathrm{y}$ sobre sus recursos presupuestarios acortando las posibilidades de avanzar hacia los objetivos del desarrollo sostenible. Es evidente que en tal contexto no se puede dejar de tomar en cuenta los desafíos no tradicionales a la seguridad. Ellos crean una zona más amplia de acción de factores destructivos, factores que minan gravemente la implementación del desarrollo sostenible. En nuestro tiempo en la región latinoamericana la pandemia de delincuencia organizada y transnacionalizada sale a primer plano entre las amenazas a la seguridad a nivel nacional y regional. Sin neutralizar dicho factor es dificil esperar el alcance de los ODS.

Al examinar la problemática de la seguridad no podemos hacer caso omiso de la otra cara de la medalla: la vulnerabilidad de la economía y de la sociedad. Aquí hay una paradoja: en nuestro mundo se perfeccionan y se ponen más complejos todos los sistemas de producción, distribución, gestión, comunicación, avituallamiento, al paso que el grado de vulnerabilidad solo va en aumento. En relación con esto tornamos a la cuestión de compleja articulación del mecanismo 
de determinación del desarrollo. La rotura de la cadena en cualquier eslabón suyo altera el equilibrio del sistema de nuestra existencia, provocando múltiples reacciones en cadena $\mathrm{y}$ bloqueando las reservas del desarrollo, hecho que lleva implícitas consecuencias catastróficas en última instancia. No hay que remontarse mucho para encontrar ejemplos al respecto. El coronavirus, a medida de la transformación de la epidemia en pandemia, nos ha dado una lección suasoria: el proceso biológico-medicinal destructivo es capaz de socavar el comercio internacional, turismo internacional, conducir a la ruptura de cadenas de producción, perturbar la navegación y el transporte aéreo transfronterizo $\mathrm{y}$, en resumidas cuentas, desestabilizar el mercado mundial de capitales, provocar distorsiones en la formación de precios, deformar, en esencia, todo el sistema de vida en los países afectados por él. Al parecer, de esta manera se pone punto final a la preparación de una crisis económica global.

El potencial de influencia de un u otro ideologema en la sociedad y la opción a base de él de su vía de desarrollo son determinados, desde luego, no tanto y no solo por la calidad de preparación teórica, ni siquiera por la presencia formal del mecanismo de implementación. El quid de la cuestión está en su aplicabilidad a las condiciones reales, en la adecuación del ideologema a las posibilidades de su percepción, a las demandas de la sociedad concreta. Y, por supuesto, el quid de la cuestión presupone la minimización de los motivos de supresión del ideologema. (Cómo no recordar aquí los costos adicionales de la "exportación de la democracia"!).

Al comentar esta circunstancia, procede prestar especial atención a un cambio sustancial de las tendencias sociales en la comunidad mundial, de hecho por dondequiera. En Europa, 
en América del Norte, en la región de Asia-Pacífico, en América Latina y el Caribe. Muchas cosas se explican y se expresan a través de la ampliación de la influencia ideológica y política de los "verdes" y la manifestación de esta tendencia en los movimientos alterglobalistas. Aún así, la cuestión reside así mismo en la ampliación de la zona de la clase media, aumentando a la vez la vulnerabilidad de su situación material y estatus social. Podemos, por supuesto, tratar de diversas maneras a Greta Thunberg. Pero, como fenómeno social ella es principalmente objetiva y no solo "moldeada por alguien".

Para los latinoamericanistas reviste especial importancia la manera en que las ideas del desarrollo sostenible se unen a la idiosincrasia de la población autóctona, en especial en medio del "renacimiento indígena" (demográfico, político y espiritual). Las creencias de los quechuas, el mayor grupo étnico autóctono del Nuevo Mundo, sirven de testimonio de su adecuación y compaginación. Se trata de una actitud sensibilísima hacia la madre tierra (pachamama), de las normas y demandas de una vida digna (sumak kawsay). En este último caso se trata del compromiso de vivir en buena armonía con la naturaleza, con la comunidad, con sí mismo. Usted dirá que no hay que ir lejos y recordará los valores del budismo y de la doctrina de Confucio. Y tendrá razón.

\section{Al valorar el sentido de la transición a la interpretación} integral de la concepción del desarrollo sostenible procede especificar que esta permite comprender la estrechez de la percepción monodisciplinaria de la realidad y la correspondiente reflexión monodisciplinaria. Tanto más es aplicable al estudio regional que, se puede decir, por definición debe ser no solo multidisciplinario sino también interdisciplinario. En esto radica su diferencia genérica de las ciencias instrumentales. A este 
respecto es oportuno recordar los resultados de la diferenciación del saber científico que ha alcanzado tal grado de "dispersión" que, afin de cuentas, lleva no solo a la profundización, profesionalización de los conocimientos, sino también a la deformación de la noción científica de complejos fenómenos y procesos, condicionados en realidad tan solo por un conjunto de factores heterogéneos y por ninguna otra cosa. Otra imagen saca el objeto en examen de su contexto real, colocándolo en "zancos" de la abstracción científica, o bien del tecnicismo analítico.

Hoy día, cuando la diferenciación de la ciencia ha alcanzado su máximo, el imperativo y la prioridad consisten, según mi criterio, en tornar al árbol de la vida que, como es sabido, verdece copiosamente. Y nos damos clara cuenta de que en la reflexión de las complejas realidades no se puede limitar a epítetos lacónicos. Hace falta, evidentemente, cierto abanico de rasgos que se promuevan a primer plano. $\mathrm{Y}$ tal tendencia (enriquecimiento de la definición) se manifiesta bastante bien en la práctica sociológica contemporánea.

Los sucesos de los últimos años nos hacen hablar de la creciente turbulencia política interna en muchos países del orbe. Con la particularidad de que esto se produce exteriorizándose en forma de impulsos periódicos y se realiza espontánea y síncronamente, extendiéndose en ocasiones a manera de epidemia. El virus de los ánimos de protesta se propaga sin parar a través de las redes sociales. Así pasó la "primavera árabe", en 2019 llegó la "primavera latinoamericana" ( $u$ otoño según las condiciones de nuestro hemisferio). Hablando de ella constatamos una sorprendente orientación heterogénea de los movimientos de protesta. Estos 

del desarrollo sostenible

vienen de derecha a izquierda y de izquierda a derecha $\mathrm{y}$, diciendo metafóricamente, "en diagonal".

Hoy día es difícil hablar de una limitación local de las olas de protesta política y social. En el deslinde del año pasado y el actual se rebelaban paralelamente las calles de Francia (París), Hong Kong, Cataluña (a favor y en contra de la secesión), Inglaterra (en pro y en contra del Brexit). Los observadores de hoy sientan involuntariamente asociaciones con lo que había ocurrido en 1968: la "primavera de Praha", el levantamiento del Barrio Latino de París, la rebelión estudiantil en México y la matanza en la Plaza Tlatelolco. Todo esto sucedió con una distancia de medio siglo y con otra coincidencia simbólica: la aparición del Club de Roma, que estaba en los orígenes del ideologema del desarrollo sostenible. Otros comentadores (entre ellos figura Kristalina Georgieva, jefa ejecutiva del FMI) tienen asociaciones de otra índole. El inicio de nuestro año, marcado con un número mágico, les impone asociaciones con aconteceres de hace una centuria. Ven allí una divergencia, disturbios políticos y espirituales, variedad de extremos: el radicalismo de izquierda y de derecha. Fue entonces cuando se reafirmó la Liga de las Naciones, cuando la "década bramante" de los años 20 había preparado la Gran Depresión. Y tras de ella vino encima la Segunda Guerra Mundial. Alusión, hablando con eufemismo, harto triste. Gracias a Dios, " La historia no se repite, pero rima", como dijo Mark Twain, citado por Kristalina Georgieva en Davos el 6 de enero de 2020.

El advenimiento de una "nueva época", cuyo sentido los expertos de la CEPAL lo ven en las consecuencias del tránsito global a raíz del cambio del paradigma tecnológico, impone la revisión de valores y añejas ideas en la zona de reflexión científica. Es obvio que la imprevisibilidad de acontecimientos 
provoca una molestia intelectual. $\mathrm{La}$ incertidumbre por su definición misma es poco sujeta a una determinación precisa. A veces se da una impresión de que la atmósfera (y la estilística) del posmodernismo se transfiere al terreno de la reflexión sociológica. Por ende, es menester aceptar el hecho de la tardanza de la reflexión. La ruptura (o incluso el vacío) que surge se llena inevitablemente con estructuras preliminares $\mathrm{o}$ bien con estructuras funcionales provisionales, lo que no se puede, desde luego, censurar, pero tampoco es posible reconocerlo como una salida óptima del aprieto.

La alusión al posmodernismo puede ser ilustrada con la intromisión de la ecléctica en el discurso teórico y con la negativa, que comenzó a menudear, al estricto diagnóstico científico. No es casual que tras la cumbre del G-20 celebrada en 2009, que puso al descubierto la confusión de razones en pos de una crisis extraordinaria, al léxico sociológico y publicístico fuera lanzada la fórmula "nueva normalidad". Paul Krugman, el Premio Nóbel, declaró al respecto, no sin sarcasmo, en su comentario para el "New York Times",con motivo de los incendios catastróficos en Australia que "el apocálipsis se presenta ahora como nueva normalidad" [3, p. 16].

Como se ha señalado, la evolución de las ideas en torno a la problemática del desarrollo sostenible avanzaba indeclinablemente en dirección a una interpretación amplificadora, pero, quizá, el giro decisorio a esta se hubiera operado a base de los resultados del trabajo de la Comisión Brundtland, en la segunda mitad de los años 80. Más adelante, el proceso de superar la interpretación netamente ecológica proseguía con "resultado in crescendo". Hoy por hoy la interpretación amplificadora se percibe como algo que de suyo se entiende. Como puntualiza James Galbraith, "ya que nos preocupa la estabilidad en el 

del desarrollo sostenible

medio ambiente, entonces hemos de preocuparnos de la estabilidad en la esfera económica. Pues aquí la inestabilidad debilita las medidas efectivas que se emprenden en respuesta a los retos globales, incluidos aquellos que tienen que ver con el cambio climático y la amenaza nuclear" [4, p. 4].

A fin de neutralizar las amenazas ecológicas, ante todo en caso de fenómenos climáticos anómalos, se necesitan y se necesitarán cuantiosos recursos financieros $y$ el asenso sin alternativa a una seria corrección del mecanis mo económico. $\mathrm{Y}$ esto significa, sin falta, el distanciamiento del confiar en la autorregulación del mercado, la renuncia a la idea fija sobre la "minimización del Estado", de sus funciones reguladoras y sus tareas de redistribución. De ello dimana la extensión de prioridades del desarrollo sostenible al medio institucional. Además, tanto a nivel nacional y regional, como al nivel global. Por lo visto, habrá que no solo modernizarla y no tanto adaptar los mecanismos existentes de regulación a las realidades cambiantes, cuanto crear instituciones y estructuras ad hoc para tareas en principio nuevas. En esto, las estructuras anteriores bien pueden emplearse como una especie de material de construcción. Y, a juzgar por la coyuntura ideológica, en la reflexión sociológica al respecto se percibe cada vez más un acento neokeynesiano.

De esta suerte, es evidente que se necesita un Estado renovado, pero este por supuesto, debe ser librado de la fatal lacra de corrupción. Lo que, a su vez, requiere dar al traste con la actividad criminal. Recordemos a propósito el colapso de residuos en Nápoles y los fracasos en su liquidación debido al entrelazamiento corrupto de intereses de los negocios logísticos criminalizados y de la administración local. Por desgracia, sobran ejemplos de esta índole. Y el problema consiste no solo 
en las posibilidades de acabar con la delincuencia (por un lado) y del saneamiento del poder público (por el otro). No hay necesidad de convencer de que se requiere cultura diferente y moral sana en la sociedad.

Alicia Bárcena, Secretaria ejecutiva de la CEPAL, afirma: si queremos garantizar el desarrollo sostenible, hace falta comprender que esto no es factible sin corregir la extrema polarización de la sociedad. "Hoy, cuando transitamos el ineludible camino hacia el desarrollo sostenible, es más urgente que nunca reafirmar que la igualdad debe ser motor del desarrollo regional y estrategia para cerrar las brechas estructurales que se han profundizado en América Latina y el Caribe" [5]. A este respecto los expertos de la CEPAL consideran que el concepto "desarrollo sostenible" resulta válido sólo si se le añade el calificativo inclusivo.

Cabe reconocer que últimamente en los debates en torno a las perspectivas del desarrollo sostenible especial atención atrae el tema de desigualdad, que es examinado cada vez más a menudo sin prevenciones políticas $\mathrm{y}$, al parecer, sin prejuicios de antaño.

¿Cuál es la situación en la distribución de los ingresos, partiendo de las estadísticas de los últimos decenios? Veamos los respectivos datos estadísticos. Es difíil negar la tendencia a la acentuación de la desigualdad en el seno de la sociedad en una serie de Estados rectores de la zona de "Occidente colectivo", en primer lugar de EE.UU. y Gran Bretaña, hecho descrito detallada y persuasivamente por el eminente estudioso británico del fenómeno de desigualdad Anthony Atkinson [6]. Por lo demás, el tema de desigualdad salió a primer plano en la ciencia sociológica mundial. Bastaría mencionar los últimos libros y artículos de Paul Krugman, Joseph Stiglitz, James 

del desarrollo sostenible

Kenneth Galbraith, las preferencias del Comité del Premio Nóbel en la elección de candidatos de los últimos años en materia de economía, los correspondientes informes de OECD, del Banco Mundial e incluso los prolijos comentarios de expertos del FMI [7]. No es casual que Kristalina Georgieva, Directora ejecutiva del FMI, al hacer un balance del año 2019, ha expresado su preocupación especial por la profundización de la desigualdad en muchos países de OECD, donde, según su parecer, esta llega a niveles sumamente altos [8]. Muy lamentablemente, Rusia también registra un nivel extremo, aproximándose a los antirécords latinoamericanos.

La dimensión social interna presupone sin alternativa alguna la atención a la estratificación social. Pues, al llegar a sus índices extremos, esta desencadena conflictos destructores que excluyen todo equilibrio, alcanzable, en principio, en vía de la evolución. A más de esto, los notables analistas que estudian la historia económica y política de EE.UU. sacan una conclusión sintomática de que la acentuación de la desigualdad económica va de la mano con la agudización de la lucha y desestabilización política. Por el contrario, la suavización de la polarización material lleva al debilitamiento del antagonismo político y a una cierta estabilización de la vida política [9, pp. 12-13]. Tal es una relación más de "causa - efecto" atinente al avance hacia el desarrollo sostenible.

Entretanto, la ampliación de la interpretación del desarrollo sostenible se ha realizado bajo un aspecto inesperado. Y un papel importante en esto ha desempeñado un personaje que antes no se inclinaba al inconformismo. El autor de la concepción del "fin de la historia" Francis Fukuyama evidentemente reconsideró su postura anterior y el "mainstream" de la sociología occidental [10]. Se pronunció 
categóricamente contra la apología de la "opción racional" que absolutiza el papel del interés económico en la toma de decisiones individuales y corporativas a cualquier nivel. En sus razonamientos Fukuyama adopta la categoría de la identidad, que se afirma cada vez más como una de las dominantes en la sociología teórica. El estudio del tema de identidad por nuestra ciencia nacional está profusamente representado por autores del Instituto de Economía Mundial y Relaciones Internacionales de la Academia de Ciencias de Rusia (ACR), que hace poco han editado una obra fundamental bajo la redacción general del miembro correspondiente de la ACR Irina S. Semenenko, obra riquísima en material teórico y en detalles de las interpretaciones [11]. Por lo demás, últimamente aparecen cada vez más publicaciones sobre este tema. Por lo que atañe a los textos de Fukuyama, en la interpretación de la identidad estos son percibidos a menudo con amplia resonancia y comprensión de la postura del autor. Quizá porque atraiga con algo personal que proviene de la controvertida búsqueda de su propia identidad de un japonés étnico nacido y criado en Chicago.

El actual material histórico le ha dado a Fukuyama abundante argumentación con respecto a que los conflictos internacionales y nacionales internos surgen en mucho e incluso por excelencia a causa del menosprecio de la identidad, sobre todo en su núcleo que para él se asocia con el sentido de la dignidad. Es de ahí de donde viene su percepción de lo que en la mentalidad de muchos pueblos se concibe como alma. No obstante, nosotros hemos de reconocer la inconclusión del estudio de dicha problemática en el cauce indicado. Y tenemos razones para corroborar esta inferencia con la competente opinión de Víctor Danílov-Danielián que permanece válida hasta la fecha: "La historia, la sociología y la psicología han 
descrito pormenorizadamente muchos aspectos de tales procesos y acontecimientos, pero no la interacción entre niveles de individuos y estructuras sociales" [12, p. 46].

Haciendo un balance de este repaso a los puntos conceptuales del desarrollo sostenible podemos colegir la siguiente fórmula. En virtud de la globalización de grandes dimensiónes y de profunda penetración, los procesos de avance progresivo de la economía, sociedad y cultura (en su sentido lato) y los sistemas de comunicación internacional se han entrelazado formando un duro nudo indisoluble. Debido a este entrelazamiento aumenta muchísimo la vulnerabilidad de todas las estructuras de base y de superestructura, lo que abre un vasto espacio para reacciones destructivas en cadena en cualquier dirección, o sea, un campo para lo que podemos calificar con toda razón de "desarrollo insostenible", refiriéndose a un modelo que hasta la fecha predomina en la práctica mundial. El peligro existencial clave radica, por una parte, en la fuerza insaciable de la militarización que pone al mundo al borde de una catástrofe nuclear $\mathrm{y}$, por la otra, en el conflicto entre los crecientes gastos de la actividad antropógena y las restricciones naturales relativas a la regeneración del entorno. Salta a la vista una paradoja sui generis: el progreso sobre la plataforma anterior (de desarrollo "insostenible") de hecho se traduce en regreso.

La antítesis relacionada con el concepto del desarrollo sostenible debe presuponer esquemáticamente lo siguiente: la nivelación de la dinámica cíclica en la economía, de las desproporciones sectoriales y territoriales; garantía de la seguridad económica nacional a base del aprovechamiento racional y responsable de los recursos materiales y financieros; superación de los vicios de la polarización material mediante el 
mejoramiento de la orientación social del desarrollo y el uso de diversos mecanismos de la redistribución de los ingresos, así como observancia de los derechos civiles fundamentales, neutralización de la corrupción y de la actividad delictiva. Pero quizá lo principal en el momento actual resida en parar el fatal deslizamiento hacia una situación irreversible en la degradación del medio ambiente. Todo esto es admisible $y$ factible únicamente en caso de que se reorganicen correspondientemente el sistema financiero, la estrategia estatal y el sistema de regulación global.

Hablando brevemente, el desarrollo sostenible será sostenible en el caso de que sea bien ponderado en lo económico, inclusivo $y$ equilibrado en lo social $y$ ecológicamente responsable, biológicamente seguro y, por fin, institucionalmente programado para observar los intereses de la mayoría y proteger los derechos de la minoría, si se supone y se garantiza la exclusión del uso de armas de exterminio en masa y la solución bélica de conflictos internacionales. Y por supuesto, en todo caso, con tal matriz de desarrollo es inadmisible, como se ha acentuado más arriba, abusar de deudas ante el futuro. Tal es, por supuesto, el ideal, mientras que la praxis es y será determinada por una aproximación mayor o menor al mismo.

\section{Bibliografía References Библиография}

1. Nikoláeva L.B. El consenso de París y cambios en la política ambiental. Iberoamérica. Moscow, núm 3, 2020, pp. 50-71.

2. Véase: Наше общее будущее. Доклад Международной Комиссии по окружающей среде и развитию (МКОСР). М., Прогресс, 1989, 376 с. [Our Common Future. Report of the World Commision on Environment and Development]. Moscow, Progress, 1989, 376 p.(In Russ.)]. 
Enfoques conceptuales para interpretar la problemática del desarrollo sostenible

3. Krugman, Paul. El apocálipsis se convierte en la nueva normalidad. Negocios. El Pais, 05.01.2020.

4. Galbraith, James K. La próxima gran transformación. Ideas. El País, 05.01.2020.

5. Bárcena, Alicia. Llegó la hora de igualdad en América Latina y el Caribe con urgencia y sin retraso. CEPAL. 6 de noviembre de 2019. Columna de opinión. Available at: https//www.cepal.org/es/artículos/2019.25.11.(accessed 15.06.2020).

6. Anthony B. Atkinson Inequality: What Can Be Done. Harvard University Press Cambridge, Massachusetts London, 2015, 384 p.

7. Bárcena, A., Prado, A. El imperativo de la igualdad. Por un desarrollo sostenible en América Latina y el Caribe. CEPAL. Buenos Aires, Ed. Siglo XXI, 2016, 245 p.; Stiglitz, J. The Great Divide. Unequal Societies and What We Can Do With Them. New York - London, 2015, 464 p.; Galbraith, J.K. Desigualdad. Lo que todo el mundo debería saber sobre la distribución de los ingresos y de la riqueza. Barcelona, Ed. Deustro, 2016, 223 p.; World Bank Group. Poverty and Shared Prosperity. Taking on Inequality. Washington D.C., 2016, 170 p.

8. Глава МВФ предупредила об угрозе новой Великой депрессии. [The head of the IMF warned of the threat of a new Great Depression (In Russ.)]. Available at: https://ria.ru/20200118/1563574338.html (accessed 20.06.2020).

9. Кругман, Пол. Кредо либерала. М., Издательство Европа, 2009, 368 c. [Krugman, Pol. Kredo liberala [Liberal creed] Moscow, Izdatelstvo Evropa, 2009, 386 p. (In Russ.)].

10. Fucuyama, Identidad. La demanda de dignidad y las políticas de resentimiento. Barcelona, Ed. DEUSTO, 2019, 206 p.

11. Идентичность: личность, общество, политика. Энциклопедическое издание. Отв. ред. И.С. Семененко. М., Весь мир, 2017, 992 c. [Identichnost': lichnost', obshestvo, politika. Entsiklopedicheskoe izdanie. Otv. Red. Semenenko I.S. [Identity: personality, society, politics. Encyclopedic edition. (Ed.): Semenenko I.S.]. Moscow, Ves'mir, 2017, 992 p. [In Russ.)].

12. Véase: Устойчивое развитие. Новые вызовы. Под ред. ДаниловаДаниэляна В.И. и Пискуловой Н.А. М., МГИМО, Аспект Пресс, 2015, 335 c. [Ustoichivoe razvitie. Novye vyzovy [Sustainable development. New challenges] (Ed.): Danilov-Danielian V.I. and Piskulova N.A. Moscow, MGIMO, Aspekt Press, 2015, 335 p. 